Huchra: What is the bolometric luminosity of the halo of NGC 4565 and how much does it contribute to the total light?

Hegyi: The halo does not contribute a significant fraction of the total light.

Tinsley: What is the luminosity of the halo and what is your estimate of its $\mathrm{M} / \mathrm{L}$ ratio?

Hegyi: All I can say is that the M/L value must be very large. It is difficult to quote a single value for the luminosity because our observations are made in a very broad wavelength band.

Ekers: It is correct that NGC 4565 does not possess a radio halo but it is also odd in that the disc emission is rather weak. I might also add that recent Westerbork HI observations disagree with the results of Krumm and Salpeter.

Zasov: Can you compare the luminosity distribution along the major and minor axis of the galaxy?

Hegyi: The particular scan paths which we have chosen are relatively insensitive to the eccentricity of halo brightness distribution. The only conclusion that may be drawn is that our observations are consistent with both a spherical halo and a slightly eccentric one.

Silk: What is the central surface brightness extrapolated from your halo fit and how does it compare with that for elliptical galaxies?

Hegyi: An extrapolation of the de Vaucouleurs $\mathrm{r}^{\frac{1}{4}}$ law to the galactic centre would yield a higher surface brightness than that of most elliptical galaxies.

Peebles: Have you tried fitting your data to a Hubble law?

Hegyi: We did not try very hard but my present impression is that the de Vaucouleurs $\mathrm{r}^{\frac{1}{4}}$ law gives a better fit.

\title{
THE SCATTER IN MASS-TO-LUMINOSITY RATIOS
}

\section{Jaan Einasto}

Mass-to-luminosity ratios of systems of galaxies as derived by different authors have a large scatter from $\sim 10$ to $\sim 200$. The observational data used by different authors differ only slightly, so the differences should lie in the treatment of the data. We have found that in most cases $<M / L>$ differences can be explained in the following ways.

1. The relative motion of companion galaxies, located at a mean distance $R$ from the main galaxy, gives the inner mass $M(R)$ of the 
system. In order to obtain the total mass the virial radius $\mathrm{R}_{\mathrm{O}}$, which is approximately 6 times larger than the mean distance of bright companions, should be used.

2. Double galaxies as well as groups of galaxies may form flat systems (as our Hypergalaxy). If this system is seen face-on, the observed velocity dispersion should be very small even if the true M/L is 1 arge.

3. If systems of galaxies are picked out in redshift space, then real systems can be split into subgroups with very small velocity dispersions which do not correspond to the real velocity dispersion of the system. On the other hand, foreground and background galaxies may increase the true velocity dispersion.

4. If groups of galaxies are embedded in a massive corona then most of the potential as well as kinetic energy of the system is concentrated in this corona. The conventional potential/kinetic energy test is not sensitive to the presence of massive coronas.

\section{DISCUSSION}

Ostriker: I would like to make a remark about the use of the words "virial discrepancy", "virial mass problem", "missing mass problem". What one does is to measure the total light from a system and determine its mass from dynamical arguments. The ratio of these numbers is the $\mathrm{M} / \mathrm{L}$ ratio. One then divides this $\mathrm{M} / \mathrm{L}$ ratio by a hypothetical value which may be thought appropriate and asks whether this ratio is unexpected or not. Since the "discrepancy" results from dividing a measurement by an assumption, this is not a real discrepancy. Masses of systems can only be determined by dynamical arguments.

Tinsley: I would like to strengthen Ostriker's comment that it is inappropriate to talk about "luminous mass" with an M/L 10 (or 5, 20, etc.) solar units. Even in ordinary populations with M/L 10, nearly all of the mass is invisible. Spectroscopic studies of galaxies show that the light is dominated by giant stars (especially in the redinfrared), whose own M/L is much less than 1 ; most of the mass in a system with $\mathrm{M} / \mathrm{L} \sim 10$ is in faint dwarfs that hardly contribute at al1 to the integrated light. Therefore, it is quite inappropriate to refer to the quantity "1uminosity x 10" as "luminous mass". I strongly agree with Ostriker's remark.

Rood: The value $\mathrm{M} / \mathrm{L}=7$ or 10 for individual galaxies is not an assumption taken out of the hat. It is based on studies of the internal motions of galaxies - stellar velocity dispersions and rotation curves.

Tremaine: One of the assumptions everyone has made is that all galaxies of a given type really do have a universal mass-to-light ratio. There seems to be no reason why the M/L values of galaxies or groups of a given type should not vary by a factor of 10 . Having said this $I$ hasten to add that I don't believe it but the possibility should be kept in 
mind, since it would resolve many of the inconsistencies in different people's results.

Jaakkola: In their standard context, the low values of $\mathrm{M} / \mathrm{L}$ and $\Omega$, obtained recent1y in some we11-known investigations, imply an "open" Universe with hardly any influence of galaxy concentrations on the Hubble flow. The picture of steady recessional motion of galaxies resembles conspicuously the mechanistic world picture of 200 years ago and, therefore, it possesses in a certain sense a metaphysical content. One can even notice a feed-back to the ancient Ptolemaic idea of "perfect motion". The usual inference about a smooth redshift-distance relation as an argument in favour of the expansion hypotheses is incorrect. The case is actually the opposite - Hubble himself considered this result as a natural consequence of a photon interaction mechanism for redshifts and he may be taken as a fair authority as regards the Hubble relation! Taking into account the fundamental role of gravitation and the absurdity of dynamics without matter, a low value of $\Omega\left(\equiv \rho_{\text {obs }} / \rho_{\text {crit }}\right)$ should be considered as a conflict between theory ( $\rho_{\text {crit }}$ ) and observations ( $\left.\rho_{\text {obs }}\right)$.

\section{STABILIZATION OF SYSTEMS OF GALAXIES BY SUBCLUSTERING}

\section{M. Ozernoy and M. Reinhardt}

Subclustering might help to solve the virial theorem paradox for systems of galaxies by hiding a major part of the potential energy in gravitationally bound subsystems. We have shown (Ozernoy and Reinhardt 1976, Astr. Astrophys., 52, 31) that even in groups of galaxies there is mass segregation, in the sense that bright group members tend to be concentrated towards the centre. Recently Wesson and Lermann (1977, Astrophys. Sp. Sci., 46, 327), realizing the importance of subclustering, proposed a quantitative method for estimating its effect on the stability of systems of galaxies. However, their assumption about the frequency of subsystems of multiplicity $n$ is not in accord with Holmberg's (1962) result. The mean frequency of galaxies in pairs is 0.37 for the Turner and Gott groups (1976) and 0.23 for the de Vauceulours groups (1976), in good agreement with the value of 0.25 required by Holmberg's distribution. Assuming Holmberg's frequency of gravitationally bound subsystems and that they are homogeneously distributed throughout the system, we have for the ratio of the total potential energy of a system of $\mathrm{N}$ equal masses $\Omega$ to the potential energy calculated in the usual way neglecting subclustering $\Omega_{\mathrm{S}}, \Omega / \Omega_{\mathrm{S}} \approx 1+\left(\mathrm{R}_{\mathrm{c}}\right) /\left(<\mathrm{r}_{2}>\mathrm{N}\right)$, if the velocity dispersion $\left\langle\sigma_{r}{ }^{2}(n)\right\rangle=$ constant. Here $R_{c}$ is the effective radius of the system and $\left\langle r_{2}\right\rangle$ the mean distance of binaries. The assumption $\sigma_{r}{ }^{2}(n)=$ const is reasonable for $n \leqslant 7$, when Holmberg's distribution holds, since $\sigma_{\mathrm{r}}{ }^{2}(2)=203 \mathrm{~km} \mathrm{~s}^{-1}$ according to Karachentsev (1974), and increases to only $\simeq 1000 \mathrm{~km} \mathrm{~s}^{-1}$ for rich clusters. Since Karachentsev's data give an $\left\langle\mathrm{r}_{2}\right\rangle=33 \mathrm{kpc}$ for $\mathrm{H}_{\mathrm{O}}=55 \mathrm{~km} \mathrm{~s}^{-1} \mathrm{Mpc}^{-1}$, we have $\Omega / \Omega_{S} \approx 4$ for groups of galaxies with $R_{c} \approx 1 \mathrm{Mpc}$ and $N=10$. Thus it seems that subclustering cannot remove the mass discrepancy for $\mathrm{rich}$ clusters and for groups only in moderate cases. 